\title{
Assessment of the Management of Post-Tonsillectomy Pain at the ENT-CFS Department of Ignace Deen National Hospital
}

\author{
Alpha Oumar Diallo ${ }^{*}$, Amadou Lamarana Diallo1, Carlos Othon Guelngar², Abdoulaye Toure ${ }^{3}$, \\ Ismael Dabo', Abdoul Aziz Diallo', Alimou Sinayoko' \\ ${ }^{1}$ ENT \& CFS Department, Ignace Deen National Hospital, Conakry, Guinea \\ ${ }^{2}$ Neurology Department, Ignace Deen National Hospital, Conakry, Guinea \\ ${ }^{3}$ Anaesthesia and Intensive Care Department, Ignace Deen National Hospital, Conakry, Guinea \\ Email: ^dalphao@hotmail.com
}

How to cite this paper: Diallo, A.O., Diallo, A.L., Guelngar, C.O., Toure, A., Dabo, I., Diallo, A.A. and Sinayoko, A. (2021) Assessment of the Management of Post-Tonsillectomy Pain at the ENT-CFS Department of Ignace Deen National Hospital. International Journal of Otolaryngology and Head \& Neck Surgery, 10, 477-486.

https://doi.org/10.4236/ijohns.2021.106042

Received: August 30, 2021

Accepted: October 11, 2021

Published: October 14, 2021

Copyright $\odot 2021$ by author(s) and Scientific Research Publishing Inc. This work is licensed under the Creative Commons Attribution International License (CC BY 4.0).

http://creativecommons.org/licenses/by/4.0/

\begin{abstract}
Introduction: Tonsillectomy is described as one of the oldest surgical procedures according to the authors of the surgical treatise (Aulus Amida and Paul of Aegina in the 6th and 7th centuries) found in the Vatican library. The contraindication of codeine in children has changed the management of post tonsillectomy pain. The aim of this study was to assess the management of post tonsillectomy pain in our developing country context. Methods: This was a prospective, analytical study lasting 6 months (September 2019-February 2020), carried out in the ENT/CFS department of the Ignace Deen National Hospital. We included in this study all patients who underwent a tonsillectomy in the department during the study period and who agreed to participate in the survey. Results: 34 patients were included in our study, i.e. a frequency of $25 \%$ of surgical interventions. The mean age of our patients was $18.06 \pm 12$ years with extremes of 03 years and 45 years. Recurrent hypertrophic tonsillitis with sleep disturbances was the most frequent indication for surgery in our study, at $47.06 \%(\mathrm{n}=16)$. We performed an isolated tonsillectomy in $52.9 \%$ $(\mathrm{n}=18)$ of cases. Analgesia was multimodal using the WHO Step I and II analgesics in $44.1 \%(n=15)$ and $55.9 \%(n=19)$, respectively. Pain control was satisfactory in all patients. Conclusion: The evaluation of post tonsillectomy pain is essential for optimal management. In this indication, multimodal analgesia, involving several levels I and II analgesics, provided satisfactory pain control.
\end{abstract}

\section{Keywords}

Tonsillectomy, Evaluation, Postoperative Pain, Conakry 


\section{Introduction}

Tonsillectomy is the surgical removal of the palatine tonsils. It is one of the most common surgical procedures performed by an ear, nose and throat (ENT) specialist [1]. It is performed on both children and adults. In North America, more than 500,000 tonsillectomies were performed in the United States in 2011 [2]. In France, Bartier S. et al. [3] on the evolution of practices in an ambulatory surgery unit showed that ambulatory tonsillectomy seems to be a preferred solution compared to conventional admission, in terms of cost savings and patient comfort, without sacrificing safety. In Burkina Faso, over a period of 9 years, tonsillectomy represented $10 \%$ of surgical procedures at the Yagaldo Ouedraogo University Hospital in Ouagadougou [1].

The indications for tonsillectomy are well defined. They concern tonsillar hypertrophy associated with sleep respiratory disorders (Obstructive Sleep Apnea/Hypopnea Syndrome), swallowing and/or phonation disorders and recurrent angina with local streptococcal, articular, cardiac and nephrological infectious complications. During this surgical procedure, postoperative pain is considered significant. It is of moderate to severe intensity, with paroxysms at the time of swallowing, caused by the mobilisation of the pharyngeal muscles and the pillars of the veil. The pain is therefore mainly inflammatory in origin and is increased by mechanical stimulation (swallowing, vomiting, etc.) and by contact with the cruciate area when food is passed through [4]. It is responsible for significant patient morbidity and potentially avoidable health costs.

For the control of this post tonsillectomy pain, different classes of analgesics are used, each with its own risk profile and unique side effects. Therefore, information on pain management should start at the pre-anaesthetic consultation, continue throughout the hospital stay and continue after the patient returns home, which is often on the same day [3].

Previously, codeine, a tier 2 analgesic, was the most prescribed drug. However, in February 2013 in the United States and April 2013 in Europe, recommendations were published by the Food and Drug Administration (FDA) and the European Medicines Agency (EMA) [5] introducing a contraindication to codeine after tonsillectomy or adenoidectomy. The aim was to optimise pain management, particularly in paediatrics, after tonsillectomy or adenoidectomy, with an alternative molecule to codeine.

The rational use of these analgesics, alone or in combination, allows post tonsillectomy pain control and ambulatory management of patients.

The objective of this study was to evaluate the post tonsillectomy analgesic management of our patients, in order to validate a management protocol adapted to our developing countries.

\section{Patient and Methods}

This was a prospective, analytical study lasting six (06) months, from September 1, 2019 to February 29, 2020, carried out in the ENT-CCF department of the Ig- 
nace Deen National Hospital (Conakry University Hospital). We included in this study all patients who underwent a tonsillectomy in the department during the study period and who agreed to participate in the survey. The analgesia protocol was developed following a review of the literature on post-tonsillectomy pain relief. The investigation was carried out as follows: The first phase consisted of the systematic administration in the operating room of paracetamol infusable at a dosage of $15 \mathrm{mg} / \mathrm{kg}$ and dexamethasone by IV at a dosage of $1 \mathrm{mg} / \mathrm{kg} / \mathrm{j}$. For subsequent analgesia, patients were divided into 2 groups: children under 15 and patients 15 years or older.

In the group of children under 15, if the pain was controlled by paracetamol infusion alone, it was maintained. If the pain was not controlled, paracetamol was replaced by Ketoprofen ( $100 \mathrm{mg}$ or $0.5 \mathrm{mg} \mathrm{kg}$ IV) which is a Non-Steroidal Anti-inflammatory Drug (NSAID). If unsuccessful, the child received a WHO Level 2 painkiller.

In the group of patients 15 years of age or older, paracetamol infusion alone was relayed by Ketoprofen (150 to $200 \mathrm{mg}$ ), paracetamol combined with codeine (300 mg/25mg) or prolonged-release Tramadol (150 at $200 \mathrm{mg} /$ day).

At six (06) postoperative hours, if the pain is well controlled, the return home is organised and the patient continues an oral analgesic treatment until D7 postoperative. Explanations are provided to the accompanying persons on how to use the pain assessment scales and how to comply with the medication. They were given a sheet with monitoring instructions to prevent overdose. Telephone monitoring was also offered. The pain rating scales used were the CHEOPS scale for children under 13, and the VAS for children 13 years or older. Controlled pain corresponded to a CHEOPS $<9$ and a VAS $<3$. Pain was assessed during swallowing, during care or at the request of the patient and/or caregivers.

The data collected on the pre-established survey forms were entered manually into the Epi Info software, version 7.2.0.1 and statistically analyzed with $p<0.05$ as the significance level. The informed consent of each patient had been obtained. We have also respected anonymity in the return of data.

The limitations of this study were the small sample size and the fact that it was mono-centric.

\section{Results}

Of the 135 surgeries performed in the department during our study, 34 involved a tonsillectomy, i.e. a proportion of $25 \%$. The mean age of our patients was 18.06 \pm 12 years with extremes of 03 years and 45 years. The 0 to 15 age group was the most represented, at $47.06 \%(n=16)$ of cases. We noted gender equality with a sex ratio of 1 (Table 1 ).

Recurrent hypertrophic tonsillitis with sleep disorders (OSAHS) was the most frequent indication for surgery in our study, i.e. $47.06 \%(\mathrm{n}=16)$ and isolated tonsillectomy was performed in 52.9\% $(\mathrm{n}=18)$ cases (Table 2).

At the end of the surgery, infusable paracetamol was systematically administered to patients and dexamethasone in $88.2 \%(n=30)$ of cases. Upon awakening, 
Table 1. Distribution of patients by socio-demographic characteristics.

\begin{tabular}{ccc}
\hline Age & Effective & Percentage \\
\hline 15 years & 16 & 47.1 \\
15 to 30 years & 15 & 44.1 \\
$>30$ years & 3 & 8.8 \\
\hline Sex & & \\
Male & 17 & 50.0 \\
Female & 17 & 50.0 \\
Total & 34 & 100.0 \\
\hline
\end{tabular}

Mean age $18.06 \pm 12$ years; Extreme 3 years and 40 years; Sex ratio $=1$.

Table 2. Distribution of tonsillectomy according to the indication and the procedures performed.

\begin{tabular}{ccc}
\hline Indications & Effective & Percentage \\
\hline Tonsillar hypertrophy with sleep disorders & 16 & 47.1 \\
Repeated angina with streptococcal complications & 10 & 29.4 \\
Tonsillar hypertrophy with swallowing disorder & 8 & 23.5 \\
\hline Gesture carried out & & \\
\hline Isolated tonsillectomy & 18 & 52.9 \\
Adeno-tonsillectomy & 15 & 44.1 \\
Tonsillectomy + Uvulectomy & 1 & 3.0 \\
Total & 34 & 100.0 \\
\hline
\end{tabular}

depending on the age of the patients, the WHO level I or II analgesics were administered as a relay. These were nonsteroidal anti-inflammatory drugs (Ketoprofen) in $44.1 \%(\mathrm{n}=15)$ of cases, Tramadol in combination with Paracetamol in $38.2 \%(n=13)$ of cases (Table 3$)$.

Digestive (nausea, vomiting) and neurological (dizziness, drowsiness) side effects associated with the use of Tramadol or Codeine have not been observed in our patients. The same is true for NSAIDs and corticosteroids. No postoperative bleeding, often responsible for reoperation, has been reported. WHO level 3 analgesics (morphine, oxycodone), reserved for pain refractory to these combinations, were not used in our study.

The assessment of pain upon awakening of children under 13 with CEOPS had reported an average of $10 \pm 7$. The subsequent assessment of pain at H6, $\mathrm{H} 12, \mathrm{H} 24, \mathrm{H} 36, \mathrm{H} 48$ and $\mathrm{H} 72$ had reported a respective mean of $6.8 \pm 1.4 ; 6.14 \pm$ 2.3; 5.2; 4.64 and 4.2 (Figure 1).

For older patients, we used EVA. This made it possible to report as the intensity of the pain at $\mathrm{H} 6, \mathrm{H} 12, \mathrm{H} 24, \mathrm{H} 36, \mathrm{H} 48$ and $\mathrm{H} 72$ on average, respectively 5.2 $\pm 1.4 ; 3.2 \pm 1.2 ; 3 ; 2.6 ; 3.2$ and 2.1 (Figure 2). 
Table 3. Distribution of tonsillectomies according to analgesics used postoperatively.

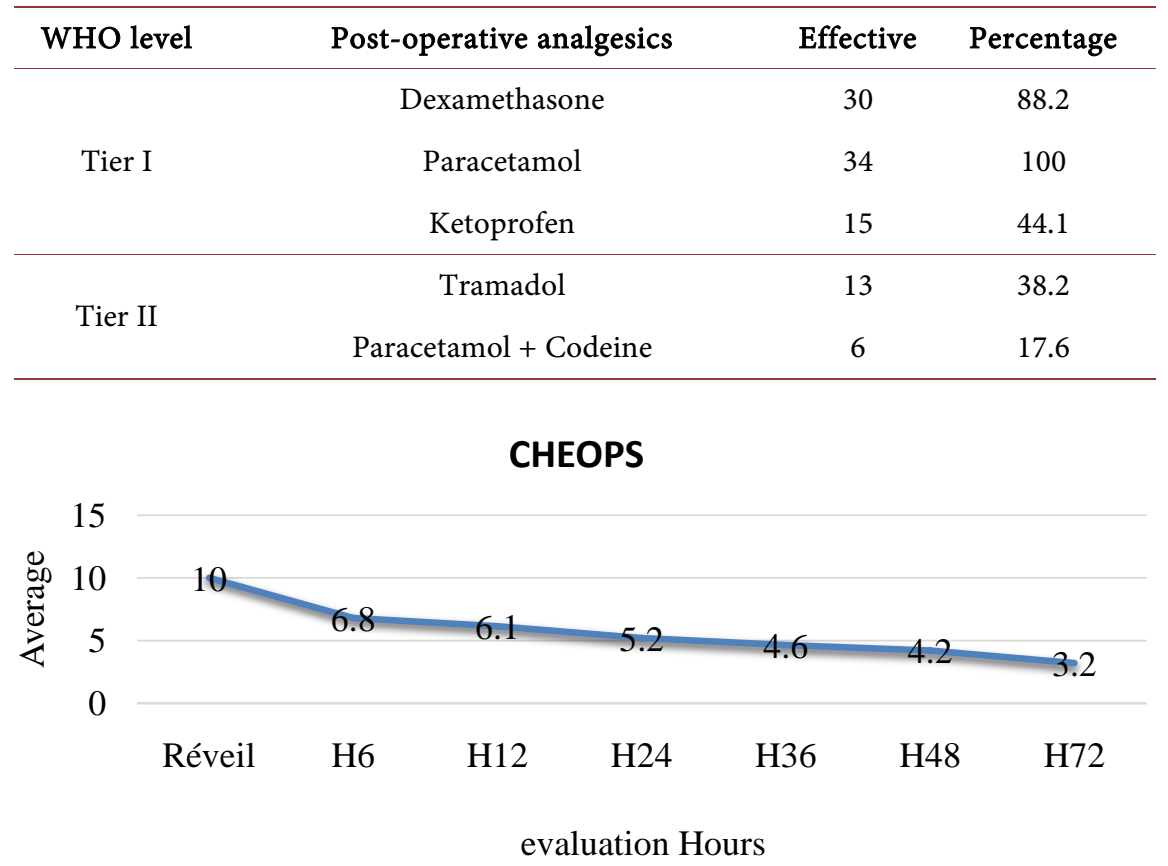

Figure 1. Assessment of pain intensity with CHEOPS.

EVA

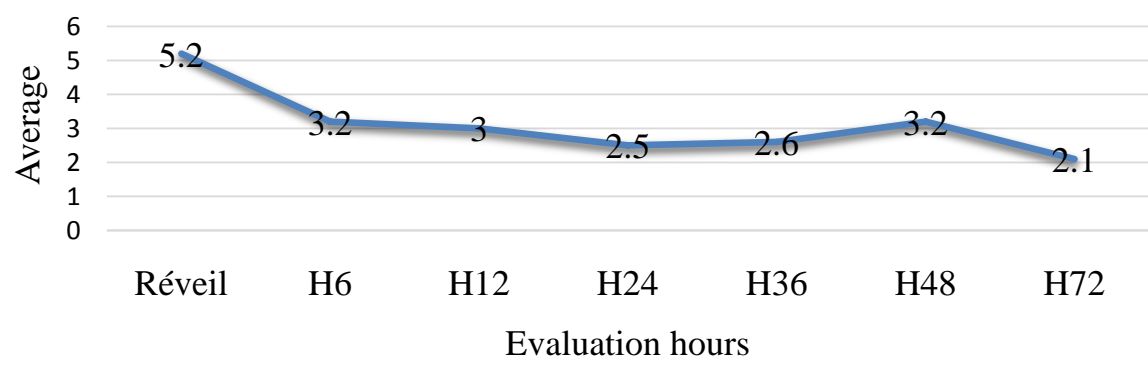

Figure 2. Assessment of pain intensity with VAS.

\section{Discussion}

We carried out a cross-sectional study in patients who had undergone tonsillectomy in our department. Indeed, this surgery is responsible for more or less intense postoperative pain. Nowadays, it is performed on an outpatient basis in most centers, sometimes outside of specific criteria for the management of postoperative pain. Its frequency varies from country to country. In the sub-region, it was $42.91 \%$ in Niamey [6] and $54.5 \%$ in Dakar [7].

The mean age of our patients was higher than that reported by Thiam N.F et al. [7] at Ouakam Military Hospital, i.e. 13.5 years with a pediatric diaper representing $63.2 \%$ of cases. This is probably related to the recurrence of this lymphoid organ hypertrophy in children more than in adults.

The correlation between gender and the indication for tonsillectomy has never been reported in the literature. However, S. Bartier et al. [3] during their study at 
the Sud Francilien hospital, noted a male predominance with a sex ratio of 2.8, which is different from our study.

The indications were numerous and dominated, in our study, by tonsillar hypertrophy resulting in sleep disorders such as obstructive sleep apnea/hypopnea syndrome (OSAHS) or $47.06 \%(n=16)$ of cases. Other indications for tonsillectomy include recurrent tonsillitis (3 to 4 infectious episodes per year over 3 consecutive years, or 5 infectious episodes over 2 years), chronic tonsillitis complications such as peritoneal abscesses or infections. Strep throat, treatment of periodic fevers (Marshall's syndrome) and biopsy for tonsil malignancy [4].

Isolated tonsillectomy was performed the most, i.e. $52.9 \%(\mathrm{n}=18)$ of our patients. It was closely followed by adeno-tonsillectomy, i.e. $44.1 \%(\mathrm{n}=15)$ of cases. The latter is of interest to the pediatric population in whom OSAHS is often associated with adenotonsillary hypertrophy. Our results are different from those reported by Burton MJ et al., [8] i.e. 86.4\% ( $\mathrm{n}=987)$ of adeno-tonsillectomy and $13.6 \%(n=156)$ of isolated tonsillectomies.

Removal of the amygdala triggers inflammatory cascades that aid healing, but also leaves an open wound in the pharynx with exposed nerve fibers and damaged muscle fibers. This combination of factors creates a postoperative wound vulnerable to mechanical trauma generated during swallowing, which increases in size during the first three postoperative days [9]. During this process, poorly controlled post-tonsillectomy pain often results in distress for both the patient and their family and can lead to dehydration due to their refusal to eat.

To better control this pain, all of our patients received paracetamol immediately after surgery. Also known as acetaminophen, paracetamol is a commonly used pain reliever and fever reducer that has been shown to be very safe for use in both children and adults and generally causes fewer side effects than drugs. Non-Steroidal Anti-Inflammatory Drugs (NSAIDs). It is commonly used as a first-line treatment for surgical pain and has been shown to be an effective analgesic for post-tonsillectomy pain [10]. However, when used alone, acetaminophen may be insufficient after tonsillectomy and is therefore often used in combination with an NSAID or an opioid to achieve adequate pain control [9]. Indeed, multimodal analgesia has become the standard of care in the management of postoperative pain. Combinations of analgesics with different modes of action are commonly used to improve analgesia and to reduce side effects.

Ketoprofen is an NSAID well known to be a potent pain reliever that works by inhibiting cyclooxygenase enzymes involved in inflammation and pain. The debate over the safety of using NSAIDs after tonsillectomy continues, in part due to the lack of powerful meta-analysis statistics to rule out the possibility that NSAIDs increase the risk of bleeding after tonsillectomy. However, the use of NSAIDs is increasingly widespread in the control of post-tonsillectomy pain and often considered as a first-line treatment in combination with acetaminophen in some settings.

Opioids are very commonly used postoperatively. They are effective in treat- 
ing acute pain. However, it is well known that they have many side effects including, but not limited to, gastrointestinal disturbances (nausea, vomiting, constipation,) respiratory depression, urinary retention, sedation and pruritus [11]. Additionally, there have been growing concerns regarding the use of codeine in children. Tramadol, also used in pain control, has risks similar to the respiratory side effects of codeine.

Dexamethasone during and after surgery is frequently used to control pain and prevent postoperative nausea and vomiting. This was the case in our study. Although caution should be exercised about the occurrence of bleeding during its use, we have not yet come across any serious prospective studies to support this hypothesis.

Regarding the measurement of the intensity of postoperative pain, we observed in our two (2) groups of patients assessed respectively by the CHEOPS and EVA scales, that the pain was significant when patients woke up after the tonsillectomy. But it was gradually brought under control with the WHO level I and II analgesics. The group of patients aged 13 and over complained more than the children. This would be linked to the fact that adults have a different pain pattern from that of children, in connection with the indications and techniques of tonsillectomy. In this segment of the population, surgery is usually performed for a chronic infection with scarred tonsils that requires dissection with coagulation, thus causing severe and lasting pain [12].

\section{Conclusion}

Assessment of post-tonsillectomy pain is essential for optimal management. The management of this post-tonsillectomy pain must balance pain control with the side effects of the respective pain relievers. In this indication, multimodal analgesia, involving several levels I and II analgesics, provided satisfactory pain control.

\section{Acknowledgements}

We thank all the authors who participated in the development of this work.

\section{Conflicts of Interest}

The authors declare no conflicts of interest regarding the publication of this paper.

\section{References}

[1] Dao, O.M., Ouédraogo, W.T., Elola, A., et al. (2006) Indications et Techniques de l'amygdalectomie dans le Service O.R.L. du CHU de Ouagadougou: A propos de 377 cas. Médecine d'Afrique Noire, 53, 320-324.

[2] Baugh, R.F., Archer, S.M., Mitchell, R.B., et al. (2011) Clinical Practice Guideline: Tonsillectomy in Children. Otolaryngology_Head and Neck Surgery, 144, S1-S30.

[3] Bartier, S., Gharzouli, I., Kiblut, N., et al. (2018) Tonsillectomy in Children and in 
Adults: Changes in Practice Following the Opening of a Day-Surgery Unit with Dedicated Operating Room. European Annals of Otorhinolaryngology, Head and Neck Diseases, 135, 301-305. https://doi.org/10.1016/j.anorl.2018.05.003

[4] Cohen, N. and Sommer, D.D. (2016) Post-Tonsillectomy Pain Control: Consensus or Controversy? Pain Management, 6, 31-37. https://doi.org/10.2217/pmt.15.58

[5] FDA (2013) FDA Drug Safety Communication: Safety Review Update of Codeine Use in Children; New Boxed Warning and Contraindication on Use after Tonsillectomy and/or Adenoidectomy.

[6] Salha, I., Abarchi, B.D., Timi, N. and Sono, A. (2018) Bilan de deux Ans d'Amygdalectomie au Service d'ORL et de Chirurgie Cervico-Faciale de l'Hôpital National de Niamey. Health Sciences and Disease, 19, 95-98.

[7] Thiam, N.F., Sy, A., Barry, M.W., Mbaye, A., Diandy, Y., Eriga, L.J.V. and Ndiaye, M. (2018) Indications, Techniques and Complications of Tonsillectomy and Adenoidectomy: A 10-Year Review at the Ouakam Military Hospital. Journal africain de chirurgie orthopédique et traumatologique, 5, 20-26.

[8] Burton, M.J., Glasziou, P.P., Chong, L.Y. and Venekamp, R.P. (2014) Tonsillectomy or Adenotonsillectomy versus Non-Surgical Treatment for Chronic/Recurrent Acute Tonsillitis. Cochrane Database of Systematic Reviews Review, 19, CD001802. https://doi.org/10.1002/14651858.CD001802.pub3

[9] Sutters, K.A. and Isaacson, G. (2014) Post-Tonsillectomy Pain in Children. American Journal of Nursing, 114, 36-42. https://doi.org/10.1097/01.NAJ.0000443769.04764.65

[10] Merry, A.F., Edwards, K.E., Ahmad, Z., et al. (2013) Randomized Comparison between the Combination of Acetaminophen and Ibuprofen and Each Constituent Alone for Analgesia Following Tonsillectomy in Children. Canadian Journal of Anesthesia, 60, 1180-1189. https://doi.org/10.1007/s12630-013-0043-3

[11] Jitpakdee, T. and Mandee, S. (2014) Strategies for Preventing Side Effects of Systemic Opioid in Postoperative Pediatric Patients. Pediatric Anesthesia, 24, 561-568. https://doi.org/10.1111/pan.12420

[12] Tolska, H.K., Hamunen, K., Takala, A., et al. (2019) Systematic Review of Analgesics and Dexamethasone for Post-Tonsillectomy Pain in Adults. British Journal of Anaesthesia, 123, e397-e411. https://doi.org/10.1016/j.bja.2019.04.063 


\section{Pre-Established Survey Form}

Form number:

Date: .../.../20

I) General information

Age: .... years.

Sex: $M$

$\mathrm{F} \square$

Profession:

II) Surgical procedure

Surgical indication:

Obstructive tonsillitis

Peritonsillar phlegmon $\square$

Anaesthesia: General

Intubation: Orotracheal $\square$

Associated procedures:

Adenoidectomy

Anaesthetic protocol:

Fluothane $\square$ Diazepam

Adjuvant: Dexamethasone $\square$

Hospital stay: One (1) day $\square$

III) Measuring pain intensity

CHEOPS scale

Recurrent tonsillitis

Halitosis $\square$

Local $\square$

Nasotracheal $\square$

Uvuloplasty $\square \quad$ Other (Specify)

Thiopental $\square \quad$ Atropine $\square$

Analgesic: Paracetamol

Two (2) days $\square \quad$ Three (3) days $\square$

\begin{tabular}{|c|c|}
\hline \multicolumn{2}{|l|}{ DAY } \\
\hline HOURS & \\
\hline \multicolumn{2}{|l|}{ CRIES } \\
\hline \multicolumn{2}{|l|}{ 1: no crying } \\
\hline \multicolumn{2}{|l|}{ 2: moaning or crying } \\
\hline \multicolumn{2}{|l|}{ 3: shrill or screaming } \\
\hline \multicolumn{2}{|l|}{ FACE } \\
\hline \multicolumn{2}{|l|}{0 : smiling } \\
\hline \multicolumn{2}{|l|}{ 1: calm, neutral face } \\
\hline \multicolumn{2}{|l|}{ 2: grimace } \\
\hline \multicolumn{2}{|l|}{ VERBAL COMPLAINTS } \\
\hline \multicolumn{2}{|l|}{0 : talks about things without complaining } \\
\hline \multicolumn{2}{|l|}{ 1: does not talk, or complains, but not of pain } \\
\hline \multicolumn{2}{|l|}{ 2: complains of pain } \\
\hline \multicolumn{2}{|l|}{ BODY (torso) } \\
\hline \multirow{2}{*}{\multicolumn{2}{|c|}{$\begin{array}{l}\text { 1: body (torso) calm, at rest } \\
\text { 2: changes position or becomes agitated, or body arched or rigid } \\
\text { or shaking, or body upright }\end{array}$}} \\
\hline & \\
\hline \multicolumn{2}{|l|}{ HANDS: touching the wound? } \\
\hline 1: does not move hand towards the wound & \\
\hline 2: moves hand forward or touches or grabs wound & \\
\hline
\end{tabular}


A. O. Diallo et al.

\section{Continued}

\section{LEGS}

1: relaxed or gentle movements

2: twisting, writhing or kicking, legs straightened or raised over body or crouching.

Total

Visual Analogue Scale (VAS)

\begin{tabular}{c|l}
\hline DAYS & \\
\hline HOURS & \\
VAS $\leq 3$ & \\
$3<$ VAS $\leq 6$ & \\
VAS $>6$ & \\
\hline
\end{tabular}

VAS $\leq 3$ (mild pain)

$3<$ VAS $\leq 6$ (moderate pain)

VAS $>6$ (severe pain)

IV) Treatment of pain:

Analgesics used:

Stage I: paracetamol: oral infusable suppository NSAID: Ibuprofen

Stage II: paracetamol codeine tramadol

Adjuvant: ice packs

Other treatments:

V) Evaluation of the effectiveness of the treatment

Are you satisfied? Yes $\square \quad$ No $\square$ 